\title{
Investigation of Intestinal Absorption Enhancers: Individual vs. Blends with the Carbamoylphosphonate JS403
}

\author{
Reut Bitton, Marina Tsuriel, R Rama Suresh, Eli Breuer, Reuven Reich and Amnon Hoffman* \\ School of Pharmacy, Institute for Drug Research, The Hebrew University of Jerusalem, 12065, Jerusalem 91120, Israel
}

\begin{abstract}
JS403 is a carbamoylphosphonate (CPO) molecule that showed anti-metastatic properties in mice. Since JS403 is intended to be a chronic prophylactic drug, the preferred route of administration should be oral. However, it exhibits poor oral bioavailability of less than $1 \%$. The poor intestinal permeability and high solubility implies its classification as BCS class III drug. The aim of this study was to overcome the limited intestinal permeation of JS403 that is regarded as an unmet need in the pharmaceutical industry for this class of drugs. Therefore, the impact of acceptable absorption enhancers on the intestinal permeability of JS403 were examined using established experimental models. The absorption enhancers were: I) sodium caprate (C10), II) sodium deoxycholate (SDC) and III) mono-carboxymethylated chitosan (MCC). The effect of each enhancer was examined alone and also in combinations. In-vitro permeability through enterocytes monolayer was studied using the Caco-2 model, while the oral bioavailability was determined by using the freely moving rat model. The results of this investigation showed that while the use of a single absorption enhancer had no effect on JS403 permeability, the combination of C10 and sodium deoxycholate increased the permeability of JS403 by 10 -fold in the in-vitro model. In addition, this blend showed a 2-fold elevation in JS403 oral bioavailability. Both in-vitro and in-vivo results highlight the synergistic potential of the combined enhancers $\mathrm{C} 10$ and sodium deoxycholate in enhancing oral bioavailability of BCS class III medications.
\end{abstract}

Keywords: Carbamoylphosphonate; Absorption enhancer; Intestinal permeability; Bioavailability; BCS Class III; Sodium caprate; Sodium deoxycholate; Chitosan; MONO-carboxymethylated chitosan

\section{Introduction}

Carbamoylphosphonates (CPOs) are small molecules [1] that share a common structure of phosphonate group linked to a sulfonamide moiety by polymethylene linker as shown in Figure 1 [2].

The diverse CPO's vary from each other in the length of the polymethylene linker, where JS403 is a CPO compound with chain of 6 methylenes and MW of $456 \mathrm{~g} / \mathrm{mol}$ [2]. We have previously demonstrated that JS403 is an inhibitor of three cancer promoting and metastasis microenvironment supporting zinc-enzymes: matrix metalloproteinase (MMP-II), carbonic anhydrase 9 and 12 (CAIX, CA-XII) and autotaxin (ATX) [3]. At physiological pH JS403 is ionized, and therefore does not penetrate into cells [2]. Thus, following administration its distribution in the body is restricted mainly to the extracellular fluids where the relevant zinc-enzymes are located. In-vivo examination of murine melanoma model where mice were injected with B16F10 cells and treated by JS403, have shown promising results of 71 percent decrease in lung metastasis following oral administration [3]. Those figures pose JS403 as a worthy candidate for anti-metastatic treatment.

The ionization of JS403 at physiological pH dictates its absorption

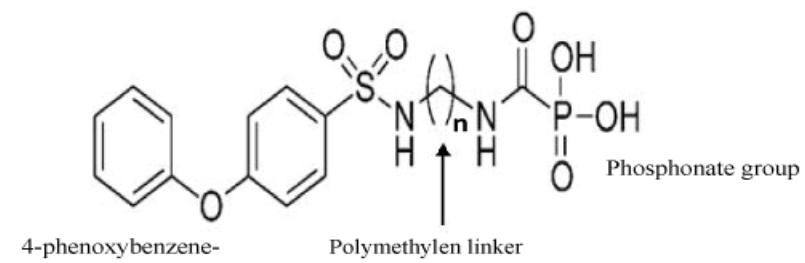

sulfonamide moiety

Figure 1: CPO structure. through paracellular pathway and therefore is responsible for its low intestinal permeability. Therefore, JS403 oral bioavailability is less than $1 \%$ [2]. Poor oral bioavailability is a stumbling difficulty when a chronic preventative anti-metastatic treatment is required. Amidon et al. have classified drugs into four groups according to their aqueous solubility and membrane permeability [4]. According to this categorization JS403 may be the candidate of BCS Class III with properties of high solubility and poor permeability. Improving oral bioavailability of BCS Class III medications is an unmet need in pharmaceutical industry. Thus, our investigation in search for enhancing the bioavailability of this $\mathrm{CPO}$ may contribute to the broader issue regarding BCS III in general.

The primary strategy to deal with low oral availability of hydrophilic drugs is by using absorption enhancer [5]. The experimental strategy in the current investigation was to evaluate the capacity of three known absorption enhancers: sodium caprate (C10), sodium deoxycholate (SDC) and mono-carboxymethylated chitosan (MCC), to affect the intestinal permeability of JS403, and compare it to the efficacy of blends of these enhancers in the same models.

Sodium caprate is a salt of a saturated fatty acid with $\mathrm{n}=10$ carbons. It is one of the most studied absorption enhancers and it is approved as a food additive and a component of a rectal suppository in Sweden and Japan [6]. Its proposed mechanism of action as absorption enhancer for hydrophilic drugs is by altering the paracellular route [7].

*Corresponding author: Amnon Hoffman, School of Pharmacy, Institute for Drug Research, The Hebrew University of Jerusalem, 12065, Jerusalem 91120, Israel, Tel: +97226757014; E-mail: amnonh@ekmd.huji.ac.i

Received February 01, 2018; Accepted February 23, 2018; Published March 06, 2018

Citation: Bitton R, Tsuriel M, Suresh RR, Breuer E, Reich R, et al. (2018) Investigation of Intestinal Absorption Enhancers: Individual vs. Blends with the Carbamoylphosphonate JS403. J Bioequiv Availab 10: 24-28. doi: 10.4172/0975-0851.1000372

Copyright: @ 2018 Bitton R, et al. This is an open-access article distributed under the terms of the Creative Commons Attribution License, which permits unrestricted use, distribution, and reproduction in any medium, provided the original author and source are credited. 
Citation: Bitton R, Tsuriel M, Suresh RR, Breuer E, Reich R, et al. (2018) Investigation of Intestinal Absorption Enhancers: Individual vs. Blends with the Carbamoylphosphonate JS403. J Bioequiv Availab 10: 24-28. doi: 10.4172/0975-0851.1000372

The paracellular pathway is transport of molecules through the intercellular spaces. It is governed by tight junctions (TJ), a dynamic structure of macromolecular assembly of multi proteins which are located at the apical side of epithelial cells and function as a regulated barrier between cells in tissues such as the intestines [8]. C10 elevate paracellular transport via complex mechanisms that include redistribution of the TJs proteins $\mathrm{ZO}-1$ and occluding, followed by additional processes that lead to increase in paracellular permeability [9].

Sodium deoxycholate (SDC) produced in the body as bile salt. In-vivo study with SDC showed improved absorption capacity of cefotaxime sodium, a hydrophilic molecule with low permeability that has been classified as BCS class III [10]. It has been reported that the absorption enhancement mechanism of action of SDC on the paracellular pathway is by loosening of the TJ [11].

Chitosan and its derivatives have also been studied for their ability to enhance intestinal permeability [12]. Chitosan is a polysaccharide derived by $\mathrm{N}$-deacetylation from the natural polymer chitin. Its ionic effect with the cell membrane enable augmentation of permeability by mucoadhesion and opening the tight junctions [12]. Chitosan is not soluble through all of physiological $\mathrm{pH}$ range and thus it cannot be used when with its natural structure [13]. Therefore, chitosan derivatives are required for use within the intestinal fluids. One such derivative is MCC that has polyampholytic character. It allows the formation of solutions even in the presence of polyanionic compounds. According to previous report, MCC showed ability to increase heparin permeability through intestine epithelial cells [14].

All the three permeability-enhancing agents that were selected in this work are presumably safe to use and seem to have potential to improve the oral bioavailability of JS403.

Our explicit aim for this study was to improve the intestinal permeability of JS403, by co-administration with the absorption enhancers: C10, SDC and MCC, each one alone and specifically their blends in order to learn whether such combinations can lead to synergistic effects. For this purpose, Caco-2 monolayer model was used first. It is accepted as standard model for assessment of intestinal transport of test molecules in in-vitro setup [15]. Subsequently, to ascertain the in-vitro findings, the preferred enhancing formulation has been investigated in-vivo in rats.

\section{Materials and Methods}

\section{Chemical synthesis}

All materials, unless otherwise stated, were purchased from SigmaAldrich (Rehovot, Israel). Mono-carboxymethyl (MCC) chitosan and JS403 were synthesized by Prof. Eli Breuer's laboratory, Institute for Drug Research, Hebrew University, Jerusalem, Israel.

\section{Caco- 2 cell monolayer assay}

Growth and maintenance of cells: Caco- 2 cells were obtained from ATCC (Manassas, VA, USA) and then grown in $75 \mathrm{~cm}^{2}$ flasks at $37^{\circ} \mathrm{C}$ in $5 \% \mathrm{CO}_{2}$ atmosphere and a relative humidity of $95 \%$. The culture growth medium consisted of Dulbecco's modified Eagle medium (DMEM) supplemented with 10\% heat-inactivated fetal bovine serum (FBS), $1 \%$ nonessential amino acids, $2 \mathrm{mM} \mathrm{L}$-glutamine, $2 \mathrm{mM}$ sodium pyruvate and $2 \mathrm{mM}$ penicillin-streptomycin solution.

Preparation of cells for transport studies: Cells in a passage range of 52-60 were seeded at a density of $25-10^{5}$ cells $/ \mathrm{cm}^{2}$ on untreated culture inserts of polycarbonate membrane with $0.4 \mu \mathrm{m}$ pores and a surface area of $1.1 \mathrm{~cm}^{2}$. The culture inserts containing the Caco-2 monolayer were placed in 24 trans-well plates $12 \mathrm{~mm}$, Co-star. The culture medium was replaced every other day. Transport studies were performed 21-23 days after seeding, when the cells were fully differentiated and the TEER values were stable (300-500 $\left.\Omega \mathrm{x} \mathrm{cm}^{2}\right)$.

Transport experiment protocol: Transport study (apical to basolateral, A to B) was initiated by medium removal from both sides of the monolayer and replacement with apical buffer $(600 \mu \mathrm{L})$ and basolateral buffer $(1500 \mu \mathrm{L})$, both warmed to $37^{\circ} \mathrm{C}$. The cells were incubated for $30 \mathrm{~min}$ at $37^{\circ} \mathrm{C}$ with shaking $(100 \mathrm{cycles} / \mathrm{min})$. Test solutions were preheated to $37^{\circ} \mathrm{C}$ and added $(600 \mu \mathrm{L})$ to the apical side of the monolayer. $50 \mu \mathrm{L}$ samples were taken from the apical side immediately at the beginning of the experiment, resulting in $550 \mu \mathrm{L}$ apical volume during the experiment. For the period of the experiment, cells were kept shaken at $37^{\circ} \mathrm{C}$. At certain predetermined times $(20$, $40,60,80,100,120$ and $150 \mathrm{~min}$ ), $200 \mu \mathrm{L}$ samples were taken from the basolateral side and replaced with the same volume of fresh basolateral buffer to maintain a constant volume.

Data analysis: The permeability coefficients (Papp) for JS403 and atenolol were calculated using the following equation:

$$
P_{\text {aap }}=\frac{\Delta Q}{\Delta t} \times \frac{1}{A \times C_{0}}
$$

where $\mathrm{Q} / \mathrm{t}$ is the steady state rate of appearance of the drug on the receiver side (obtained from the linear plot of drug accumulated $v s$. time), $\mathrm{C}_{0}$ is the initial concentration of the drug on the donor side, and $A$ is the surface area available for permeation [16].

\section{In vivo pharmacokinetic studies}

Male Wistar rats (275 - 300 g) were purchased from Harlan laboratories (Rehovot, Israel). All rats receive free access to water and food up to three hours before the experiment. All surgical and experimental procedures were reviewed and approved by the Animal Experimentation Ethics Committee of the Hebrew University Hadassah Medical Center, Jerusalem. In order to enable convenient blood sampling, an indwelling cannula was implanted into the right jugular vein 24 hours before the pharmacokinetic experiment as described before [17]. During the cannula implantation rats were anesthetized by intra-peritoneal injection of $1 \mathrm{~mL} / \mathrm{kg}$ of ketamine-xylazine solution (9:1, respectively). The cannula was tunnelled beneath the skin and exteriorized at the dorsal part of the neck. After completion of the surgical procedure, the animals were kept overnight in individual cages. Four hours post oral administration free access to food and water was available. Animals were randomly assigned to different experimental groups. JS403 was administered IV through the implanted cannula or PO using oral gavage. The IV JS403 dose was $30 \mathrm{mg} / \mathrm{kg}$ in $1 \mathrm{ml} /$ $\mathrm{kg}$ solution and for the oral formulation JS403 solution $(30 \mathrm{mg} / \mathrm{ml})$ was prepared with $300 \mathrm{mg} / \mathrm{kg}$ JS403 dose and $100 \mathrm{mg} / \mathrm{kg}$ dose for C10, sodium deoxycholate [18]. The solution was freshly prepared prior to administration. Dosing blood samples of $300 \mu \mathrm{L}$ were taken at $0,0.05$, $0.5,1,3,7,12,24,36$ and $48 \mathrm{~h}$ post-dosing for IV and at $0,0.16,0.5,1$, $3,6,10,24,36$, and $48 \mathrm{~h}$ for PO. Equal volumes of heparinized saline were administered after each blood sampling. Plasma samples were separated by centrifugation $\left(4 \mathrm{~g}, 10 \mathrm{~min}\right.$, and $\left.4^{\circ} \mathrm{C}\right)$ and stored at $-20^{\circ} \mathrm{C}$ pending analysis.

\section{Analytical methods}

$150 \mu \mathrm{L}$ of plasma were mixed with $15 \mu \mathrm{L}$ of the internal standard 
Citation: Bitton R, Tsuriel M, Suresh RR, Breuer E, Reich R, et al. (2018) Investigation of Intestinal Absorption Enhancers: Individual vs. Blends with the Carbamoylphosphonate JS403. J Bioequiv Availab 10: 24-28. doi: 10.4172/0975-0851.1000372

TCH18, a CPO compound with a linker of seven methylenes. Then, $285 \mu \mathrm{L}$ of methanol were added and vortex-mixed for $1 \mathrm{~min}$. After centrifugation at $10000 \mathrm{rpm}$ for $10 \mathrm{~min}$, the organic layer was transferred to fresh glass test tubes, filtered and evaporated to dryness (Vacuum Evaporation System, Labconco, Kansas City, MO) and reconstituted in $80 \mu \mathrm{L}$ of mobile phase. JS403 amount was determined by using a high-performance liquid chromatography (HPLC) system (Waters 2695 Separation Module) with a mass-spectrometer (Waters Micro-mass ZQ, Waters Corporation, Milford, MA). The HPLC-MS conditions were as follows: Xterra MS C18, $3.5 \mu \mathrm{m}, 100 \mathrm{~A}, 100 \times 2.1$ $\mathrm{mm}$ column (Waters), an isocratic mobile phase, methanol: water: ammonium formate buffer in water $50 \mathrm{mM}$ titrated with formic acid to $\mathrm{pH}-3.8-3.9(57: 23: 20 \mathrm{v} / \mathrm{v} / \mathrm{v})$, flow rate of $0.2 \mathrm{~mL} / \mathrm{min}$ at $45^{\circ} \mathrm{C}$.

\section{Pharmacokinetic (PK) analysis}

To determine PK parameters area under the plasma concentrationtime curve (AUC) was calculated by using the trapezoidal rule.

\section{Statistical analysis}

All values are expressed as mean \pm standard deviation mean (SD). To determine statistically significant differences among the experimental groups, t-test or one-way ANOVA, followed by Tukey's test, was used. A p value less than 0.05 were termed significant.

\section{Results}

\section{In vitro Transport Studies}

Caco- 2 cells monolayer were used to assess the effect of C10, SDC and MCC as single or blends on JS403 permeability. The permeability was determined by comparing the permeability constant (Papp) of JS403 with and without the absorption enhancers. Atenolol served as positive control for paracellular permeability [19]. Although its oral bioavailability higher than expected, it is at the high range of the low permeability.

The effect of single absorption enhancer: C10, sodium deoxycholate and MCC on JS403 permeability.

The Papp values obtained following co-administration of JS403 with single enhancer is summarized in Figure 2. The statistical analysis showed no significant differences in JS403 Papp value with or without enhancer.

Effect of combined absorption enhancers on JS403 permeability in Caco-2 model: The permeability enhancing effect of the following binary combinations: (1) C10+MCC, (2) SDC +MCC and (3)

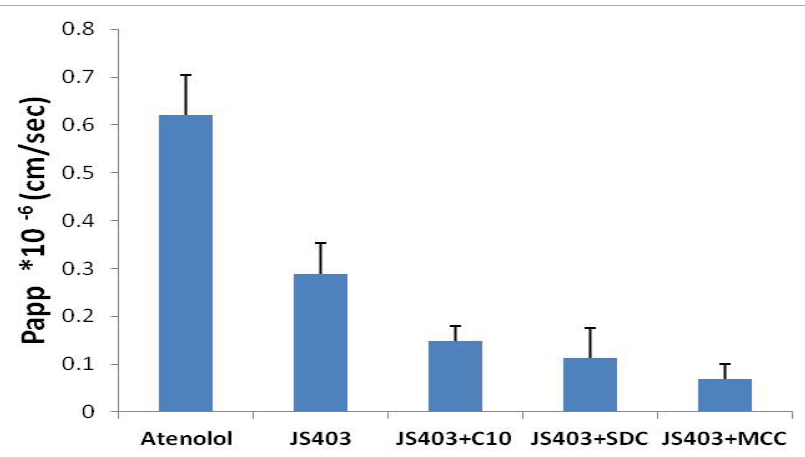

Figure 2: JS403 permeation through Caco-2 cells with or without the presence of C10, SDC and MCC $(n=3)$. Concentrations of C10 and SDC were $0.02 \% w / v$ and $0.01 \%$ for MCC.
C10+ SDC and combination of the three of them together on JS403 permeability is depicted in Figure 3. The MCC+ C10, MCC+ SDC and $\mathrm{C} 10+\mathrm{SDC}+\mathrm{MCC}$ combinations did not elevate the permeation of JS403 through Caco-2 cells. However, the combination of C10 with SDC resulted with 10-fold increase in Papp of JS403 as shown in Figure 3.

\section{In-vivo absorption study}

In light of the in-vitro results, the impact of the preferred combination C10 and SDC on JS403 oral bioavailability was examined. The JS403 serum concentration $v s$. time following single oral administration of JS403 at dose of $300 \mathrm{mg} / \mathrm{kg}$ with or without C10 and SDC at $100 \mathrm{mg} / \mathrm{kg}$ dose is showed in Figure 4 . In agreement with the in-vitro results, co-administration of JS403 with this blend solution resulted in 2-fold increase in AUC as presented in Table 1.

In addition, the semi log presentation of JS403 PK data compared to intravenous administration has been added in Figure 5 showing similarity between the three elimination graphs.

\section{Discussion}

JS403 is a promising candidate for the prevention of recurring cancer metastasis. Therefore, following of eradication of solid tumour by surgery or chemotherapy, to prevent recurrent episodes, it should be administered for a lifetime. For this reason, it is very important to assure the patient's convenience and good compliance via oral

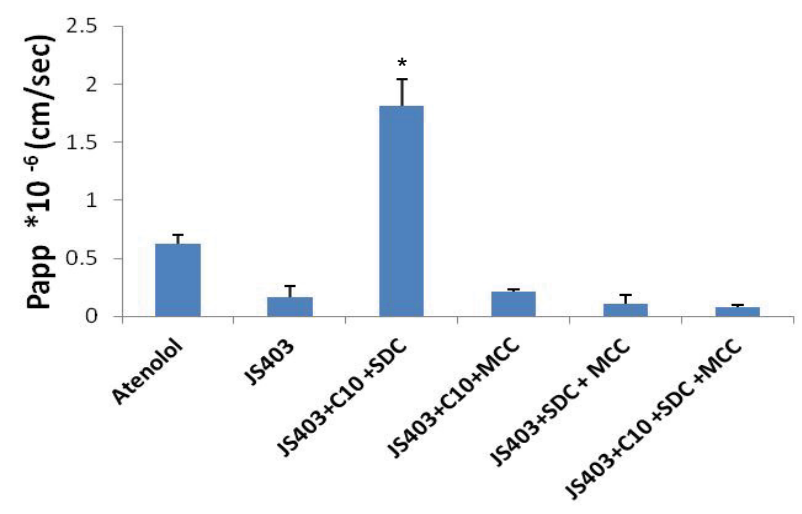

Figure 3: JS403 permeation through Caco-2 cells with or without the blends of C10, SDC and MCC $(n=3)$. Concentrations of $\mathrm{C} 10$ and sodium deoxycholate were $0.02 \% \mathrm{w} / \mathrm{v}$ and $0.01 \%$ for MCC $\left(^{*}\right)$ A significant difference from JS403 $(p<0.05)$.

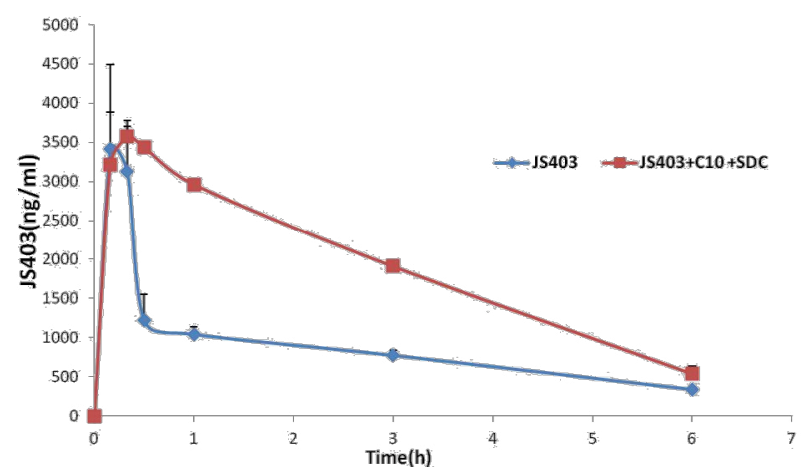

Figure 4: JS403 plasma concentration versus time curve following single ora administration of JS403 compared to single oral co-administration of JS403, C10 and SDC to rats $(\mathrm{n}=3)$. JS403 $300 \mathrm{mg} / \mathrm{kg}$, C10 and SDC $100 \mathrm{mg} / \mathrm{kg}$. 
Citation: Bitton R, Tsuriel M, Suresh RR, Breuer E, Reich R, et al. (2018) Investigation of Intestinal Absorption Enhancers: Individual vs. Blends with the Carbamoylphosphonate JS403. J Bioequiv Availab 10: 24-28. doi: 10.4172/0975-0851.1000372

\begin{tabular}{|c|c|c|c|c|}
\hline & $\begin{array}{c}A^{A U C_{0-6}} \\
\left(h^{*} n g / m L\right)\end{array}$ & $\underset{(\mathrm{ng} / \mathrm{mL})}{\mathrm{C}_{\max }}$ & $\begin{array}{l}T_{\max } \\
\text { (h) }\end{array}$ & $\begin{array}{l}\mathbf{t}_{1 / 2} \\
\text { (h) }\end{array}$ \\
\hline JS403 (iv) & $146488 \pm 56904$ & & & $1.57 \pm 0.54$ \\
\hline JS403 (po) & $5460 \pm 591$ & $3415 \pm 1527$ & 0.16 & $2.37 \pm 0.68$ \\
\hline $\begin{array}{l}\text { JS403+ } \\
\text { C10 and } \\
\text { SDC (po) }\end{array}$ & $11586 \pm 256\left(^{*}\right)$ & $3574 \pm 284$ & 0.33 & $1.97 \pm 0.27$ \\
\hline
\end{tabular}

Table 1: AUC, $C_{\max }, T_{\max }$ and $t_{12}$ values (mean $\pm S D$ ) obtained following single administration of JS403 IV at dose of $30 \mathrm{mg} / \mathrm{kg}$ compared to single oral adminstration of JS403 at dose of $300 \mathrm{mg} / \mathrm{kg}$ with or without C10 and SDC at dose of $100 \mathrm{mg} / \mathrm{kg}$ $(n=3)\left(^{*}\right)$ A significant difference in AUC $(p<0.05)$.

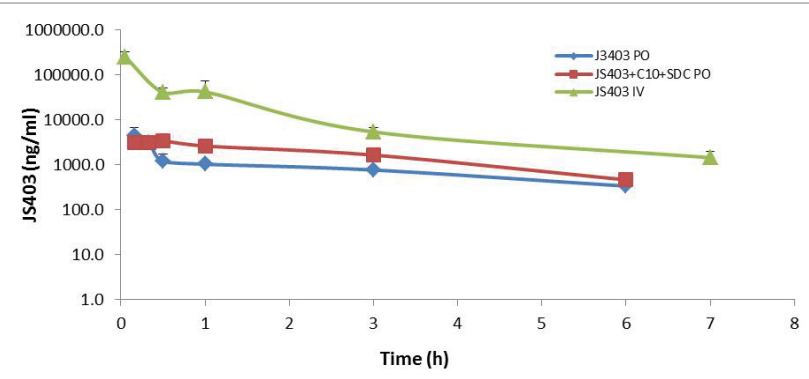

Figure 5: A semi-logarithmic plot of plasma concentration time profiles in rats for single administration of JS403 iv at dose of $30 \mathrm{mg} / \mathrm{kg}$ compared to single oral administration of JS403 at dose of $300 \mathrm{mg} / \mathrm{kg}$ with or without C10 and SDC at dose of $100 \mathrm{mg} / \mathrm{kg}$ each one. ( $\mathrm{n}=3$ for each group).

administration [20]. The poor oral bioavailability of less than $1 \%$ of JS403 is considered as an obstacle. Thus, the aim of this study was to find suitable absorption enhancers and particularly their blends for designing a suitable pharmaceutical formulation. The findings may lead also for a more generic solution that would be a suitable formulation for other BCS class III compounds that are hydrophilic drugs, highly soluble but poorly permeable thorough the intestinal membrane [4]. Many BCS class III compounds are not used clinically due to the permeability limitation. Thus, improving JS403 permeability is a major academic and pharmaceutical industry challenge.

For this purpose, we performed in-vitro studies using the Caco2 model. These studies were conducted with intention to inspect the impact of the absorption enhancers on JS403 permeability. To verify the validity of the in-vitro study, atenolol served as reference standard being positive control of paracellular pass-way transport mechanism with Papp of $6.21 \mathrm{E}-07$ ( \pm 0.83E-07).

Previously reported data demonstrated that all three absorption enhancers presented, were shown to increase permeability of certain medications [21-24]. Based on these reports JS403 permeability following co-administration with either one of the enhancers or their blend was tested. When the drug is tested with each of the enhancers separately, they fail to improve permeability (Figure 2). The selected concentrations that were used were relatively low to minimize possible vulnerability of intactness or cell vitality, and with the intention of being clinically relevant. The selected concentrations were shown before to serve as proper absorption enhancers by affecting TJ. The results of the current investigation clearly demonstrate lack of effect on the JS403 Papp. These results followed the underlying hypothesis of this investigation that a blend of such enhancers, that affect $\mathrm{TJ}$ by different mechanisms, may have a synergistic effect. The impact of all possible combinations on the JS403 Papp was inspected, including: C10 + SDC, $\mathrm{C} 10+\mathrm{MCC}, \mathrm{SDC}+\mathrm{MCC}$ and $\mathrm{C} 10+\mathrm{SDC}+\mathrm{MCC}$. The corresponding Papp values were $1.81 \pm 0.23,0.22 \pm 0.01,0.11 \pm 0.07$ and $0.08 \pm 0.02$ respectively as summarized in Figure 3. Of all the examined blends, the one that led to a significantly elevation in permeability was $\mathrm{C} 10+$ SDC, a binary combination that resulted in a 10-fold increase of JS403 permeability, apparently by a synergistic effect.

In light of these finding, this preferred binary combination that provided high effectiveness in the in-vitro enterocytes monolayer was examined in the freely moving rat model.

This model enables to effectively evaluate the intestinal absorption mechanisms involved in the input of drugs from the gut lumen into the systemic circulation. It enables also to characterize the PK profile of the drug's appearance in the blood. The in-vivo doses selection was based on a previous study which examined the effect of C10 and SDC separately in in vivo system and showed that each enhancer separately improved the gastrointestinal absorption of berberine chloride. The conclusion of this study group was to test the combined administration of these two absorption coefficients as we did in this study [18]. Our results of the in vivo experiment in rats show that $\mathrm{C} 10$ and SDC blend increased bioavailability, thus corroborated our in-vitro results (Figure 4). Namely, the selected enhancer pairing increased the AUC of JS403 by 2 folds. The terminal slope of the two oral formulations (representing the elimination phase) where comparable to that seen following bolus intravenous administration, as expected (Figure 5)

Although there is a difference in the proportion of enhancement, there is a similar trend of improvement in JS403 permeability for both in-vitro and in vivo outcomes. This difference is attributed to the fact that the in-vitro model does not fully simulate the physiological processes that occur in the intestinal fluids and other first pass mechanisms which affect the molecule's absorption from the gastrointestinal tract into the systemic blood circulation. The findings reinforce the necessity of in-vivo experiments to verify the in-vitro findings.

\section{Conclusion}

Our hypothesis that combination of permeability enhancers may have synergistic effect was indeed validated. The findings highlight the power of absorption enhancers blends as the preferred formulation for enhancing oral bioavailability of BCS class III compounds. In order to enhance the oral administration of JS403, the findings of the current investigation should be extended seeking for an optimal ratio between the enhancers in order to have the appropriate blend of C10 and SDC with JS403.

\section{Acknowledgments}

This work was mainly supported by NOFAR grant 53302 of the Chief Scientist Ministry of Industry and Commerce, Israel. A Hoffman R. Reich and E. Breuer are affiliated with the R Bloom Center for Pharmacy, School of Pharmacy the Hebrew University of Jerusalem, Israel.

\section{References}

1. Ohlmeyer M, Zhou MM (2011) Integration of small molecule discovery in academic biomedical research. Mt Sinai J Med 350-357.

2. Frant J, Veerendhar A, Chernilovsky T, Nedvetzki S, Vaksman O, et al. (2011) Orally active, antimetastatic, nontoxic diphenyl ether-derived carbamoylphosphonate matrix metalloproteinase inhibitors. Chem Med Chem 6: 1471-1477.

3. ReichR, Hoffman A, Suresh R, Shai O, Frant J, etal. (2015)Carbamoylphosphonates inhibit autotaxin and metastasis formation in vivo. J Enzyme Inhib Med Chem 30: 767-772.

4. Shah V, Amidon GL (2014) A theoretical basis for a biopharmaceutic drug classification: The correlation of In Vitro drug product dissolution and In Vivo bioavailability. Pharm Res 12: 413-420.

5. Gomez-Orellana I (2005) Strategies to improve oral drug bioavailability. Expert Opin Drug Deliv 2: 419-433. 
Citation: Bitton R, Tsuriel M, Suresh RR, Breuer E, Reich R, et al. (2018) Investigation of Intestinal Absorption Enhancers: Individual vs. Blends with the Carbamoylphosphonate JS403. J Bioequiv Availab 10: 24-28. doi: 10.4172/0975-0851.1000372

6. Maher S, Leonard TW, Jacobsen J, Brayden DJ (2009) Safety and efficacy of sodium caprate in promoting oral drug absorption: From in vitro to the clinic. Adv. Drug Deliv 61: 1427-1449.

7. Berglind E, Thórarinn G, Ólafur B, Már M (2014) N-alkylation of highly quaternized chitosan derivatives affects the paracellular permeation enhancement in bronchial epithelia in vitro. Eur J Pharm Biopharm 86: 55-63.

8. Salama NN, Eddington ND, Fasano A (2006) Tight junction modulation and its relationship to drug delivery, Adv Drug Deliv Rev 58: 15-28.

9. Lindmark T, Schipper N, Lazorová L, de Boer AG, Artursson P (1998) Absorption enhancement in intestinal epithelial caco-2 monolayers by sodium caprate: Assessment of molecular weight dependence and demonstration of transport routes. J Drug Target 5: 215-223.

10. Sharma P, Varma MVS, Chawla HPS, Panchagnula R (2005) In situ and in vivo efficacy of peroral absorption enhancers in rats and correlation to in vitro mechanistic studies. Farmaco 60: 874-883.

11. Sakai M, Imai T, Ohtake H, Azuma H, Otagir M (1997) Effects of absorption enhancers on the transport of model compounds in Caco-2 cell monolayers: Assessment by confocal laser scanning microscopy. J Pharm Sci 86: 779-785.

12. Renukuntla J, Vadlapudi AD, Patel A, Boddu SHS, Mitra AK (2009) Approaches for Enhancing Oral Bioavailability of Peptides and Proteins 447: 1841-1850.

13. Mourya VK, Inamdar NN (2009) Trimethyl chitosan and its applications in drug delivery. J Mater Sci Mater Med 20: 1057-1079.

14. Thanou M, Verhoef JC, Junginger HE (2001) Oral drug absorption enhancement by chitosan and its derivatives. Adv Drug Deliv Rev 52: 117-126.

15. Ingels FM, Augustijns PF (2003) Biological, Pharmaceutical, and Analytical Considerations with Respect to the Transport Media Used in the Absorption Screening System, Caco-2. J Pharm Sci 92: 1545-1558.

16. Ovadia O, Greenberg S, Chatterjee J, Laufer B, Opperer F et al. (2011) The effect of multiple $\mathrm{N}$-methylation on intestinal permeability of cyclic hexapeptides Mol Pharm 82: 479-487.

17. Hoffman A, Levy G (1989) Kinetics of drug action in disease states. XXIX. Effect of experimental nephrotic syndrome on the pharmacodynamics of heptabarbital: implications of severe hypoalbuminemia. J Pharmacol Exp Ther 249: $117-122$

18. Fan D, Wu X, Dong W, Sun W, Li J, et al. (2013) Enhancement by sodium caprate and sodium deoxycholate of the gastrointestinal absorption of berberine chloride in rats. Drug Dev. Ind Pharm 39: 1447-1456.

19. Yin J, Duan H, Shirasaka Y, Prasad B, Wang J (2015) Atenolol renal secretion is mediated by human organic cation transporter 2 and multidrug and toxin extrusion proteins. Drug Metab Dispos 43: 1872-1881.

20. Sastry S, Nyshadham J, Fix J (2000) Recent technological advances in oral drug delivery - a review. Pharm Sci Technolo Today 3: 138-145.

21. Mori S, Matsuura A, Rama PYV, Takada K (2004) Studies on the intestinal absorption of low molecular weight heparin using saturated fatty acids and their derivatives as an absorption enhancer in rats. Biol Pharm Bull.

22. Sugiyama T, Yamamoto A, Kawabe Y, Uchiyama T, Quan YS, et al. (1997) Effects of various absorption enhancers on the intestinal absorption of water soluble drugs by in vitro using chamber method: Correlation with an in situ absorption experiment. Biol Pharm Bull.

23. Thanou M, Nihot MT, Jansen M, Verhoef J, Junginger CHE (2001) Mono$\mathrm{N}$-carboxymethyl chitosan (MCC), a polyampholytic chitosan derivative, enhances the intestinal absorption of low molecular weight heparin across intestinal epithelia in vitro and in vivo. J Pharm Sci.

24. Brayden DJ, Walsh E (2014) Efficacious intestinal permeation enhancement induced by the sodium salt of 10 -undecylenic acid, A medium chain fatty acid derivative. AAPSJ. 